\title{
On covariant actions for chiral $p$-forms
}

\author{
Karapet Mkrtchyan ${ }^{1}$ \\ Max Planck Institute for Gravitational Physics (Albert Einstein Institute), \\ Am Mühlenberg 1, 14476 Potsdam, Germany \\ E-mail: karapet.mkrtchyan@aei.mpg.de
}

ABStRaCT: We construct a Lorentz and generally covariant, polynomial action for free chiral $p$-forms, classically equivalent to the Pasti-Sorokin-Tonin (PST) formulation. The minimal set up requires introducing an auxiliary $p$-form on top of the physical gauge $p$ form and the PST scalar. The action enjoys multiple duality symmetries, including those that exchange the roles of physical and auxiliary $p$-form fields. Same type of actions are available for duality-symmetric formulations, which is demonstrated on the example of electromagnetic field in four dimensions. There, the degrees of freedom of a single Maxwell field are described employing four distinct vector gauge fields and a scalar field.

KeYwords: Duality in Gauge Field Theories, Field Theories in Higher Dimensions, Gauge Symmetry

ArXiv EPrint: 1908.01789

To my mother

\footnotetext{
${ }^{1}$ E-mail: karapet.mkrtchyan@sns.it
} 


\section{Contents}

1 Introduction 1

2 Polynomial action for free chiral $p$-forms 2

2.1 Equivalence to PST 3

2.2 Manifestation of two abelian gauge symmetries 4

3 Chiral two-form in six dimensions 5

$\begin{array}{lll}4 & \text { Chiral boson in two dimensions } & 7\end{array}$

5 Duality-symmetric electromagnetism in four dimensions $\quad 8$

6 Conclusions 9

$\begin{array}{ll}\text { A Abelian chiral two-forms in six dimensions } & 10\end{array}$

B (Non-)chiral scalar in two dimensions $\quad 13$

$\begin{array}{ll}\text { B.1 A generalisation } & 13\end{array}$

B.2 A duality-symmetric formulation for 2d scalar 13

\section{Introduction}

The progress in our understanding of a given theoretical framework often happens when we tackle the specific "marginal" examples where the framework fails in its standard form. In Field Theory, a problem of this kind is related to chiral $p$-forms and their interactions, also related to the problem of manifesting (electric-magnetic) duality in familiar field theories.

Duality-symmetric formulations and chiral $p$-forms have been studied to great extent in the past forty years (see [1-30] for a non-comprehensive list of key references). It is known that the construction of space-time covariant actions for free chiral p-forms requires the use of auxiliary fields. There are several formulations which deal with this issue. The most economic and efficient one is that of PST $[15,16,20]$. It uses a single auxiliary scalar field which however enters the action (under derivatives) in a non-polynomial way. In this paper we will provide a simple polynomial action which contains more auxiliary fields and is classically equivalent to that of PST. It combines manifest Lorentz covariance, finite number of fields, polynomial form, no ghosts and consistence with general covariance.

Exploring alternative formulations for free field theories may open new possibilities for their interacting extensions. The simplest example to demonstrate this is the familiar scalar field with its $(d-2)$-form gauge field dual (so-called "notoph" [31]). While for a scalar field one can immediately formulate interacting theories with arbitrary non-derivative 
interaction potential, the dual formulation via $(d-2)$-form gauge field does not admit nonderivative interactions. Therefore, if we were to start with the $(d-2)-$ form, we would not be able to construct, e.g., the massless scalar field theory with $\phi^{4}$ interaction. The long-standing problem of non-abelian interactions for chiral $p$-forms motivates the search for a suitable formulation of the free theory, which will allow for non-abelian extensions.

Recently, inspired by String Field Theory, Ashoke Sen devised an action [32, 33] for chiral $p$-forms, that has all of the aforementioned virtues except for general covariance. Even though it contains an extra ghost, the latter decouples from the physical sector, therefore allowing for unitary dynamics of the physical degrees of freedom. The field variable, containing the chiral $p$-form degrees of freedom for the Sen's model is the $(p+1)$ form field strength. Possible non-abelian extensions for chiral $p$-forms would likely prefer potentials as basic variables instead, similarly to what happens in Yang-Mills theory. It is indeed only possible to write Born-Infeld type interactions through curvatures, while the minimal couplings of Yang-Mills type are available only in terms of gauge-variant potentials.

The action we derive here has all of the properties listed above, and is written through gauge potentials, therefore is a good starting point in the search for an interacting extension that would describe non-abelian chiral $p$-form theories.

The formulation we study here applies also to duality-symmetric theories. We will show it on the example of Maxwell theory in four dimensions in section 5.

\section{Polynomial action for free chiral $p$-forms}

We start with a Lagrangian for a chiral $p$-form $\varphi_{\mu_{1} \ldots \mu_{p}}$ in $d=2 p+2$ dimensions: $^{1}$

$$
\begin{aligned}
\mathcal{L}= & -\frac{1}{2(p+1)} F_{\mu_{1} \ldots \mu_{p+1}} F^{\mu_{1} \ldots \mu_{p+1}}-\frac{1}{2(p+1)}\left(\mathcal{F}_{\mu_{1} \ldots \mu_{p+1}}-(p+1) c_{\left[\mu_{1}\right.} R_{\left.\mu_{2} \ldots \mu_{p+1}\right]}\right) \\
& \times\left(\mathcal{F}^{\mu_{1} \ldots \mu_{p+1}}-(p+1) c^{\left[\mu_{1}\right.} R^{\left.\mu_{2} \ldots \mu_{p+1}\right]}\right)+G^{\mu \nu} \partial_{[\mu} c_{\nu]}
\end{aligned}
$$

where

$$
\begin{aligned}
& F_{\mu_{1} \ldots \mu_{p+1}}=(p+1) \partial_{\left[\mu_{1}\right.} \varphi_{\left.\mu_{2} \ldots \mu_{p+1}\right]}, \\
& \mathcal{F}_{\mu_{1} \ldots \mu_{p+1}}=F_{\mu_{1} \ldots \mu_{p+1}}+\frac{1}{(p+1) !} \epsilon_{\mu_{1} \ldots \mu_{p+1} \nu_{1} \ldots \nu_{p+1}} F^{\nu_{1} \ldots \nu_{p+1}},
\end{aligned}
$$

while $c_{\mu}, R_{\mu_{1} \ldots \mu_{p}}$ and $G^{\mu \nu}$ are auxiliary fields with fully antisymmetric set of Lorentz indices. Even though the Lagrangian given above is not quadratic in fields, it is quadratic in the physical gauge potential $\varphi_{\mu_{1} \ldots \mu_{p}}$ and can be shown to describe exactly a single chiral degree of freedom, in Minkowski space of $2 p+2$ dimensions for even ${ }^{2} p$.

\footnotetext{
${ }^{1}$ For chiral forms, we take $p$ to be even. A similar action will be discussed in the following for dualitysymmetric odd-forms which require doubled set of fields. For odd $p$, the chiral fields do not exist in Minkowski background. The regime of validity of our formulation is the same as that of the PST formalism - it is only available in Minkowski signature. We thank Dmitri Sorokin for correspondence on this matter.

${ }^{2}$ For even $p$ the chiral (self-dual) fields are non-trivial in Minkowski spaces and for odd $p$ - in Euclidean spaces. We will not discuss other possible signatures except for Minkowski here. We choose mostly plus signature convention for the metric.
} 


\subsection{Equivalence to PST}

We will first show that (2.1) describes a free chiral $p$-form in $d=2 p+2$ dimensions. For that, we can integrate out the auxiliary field $R_{\mu_{1} \ldots \mu_{p+1}}$ and end up with an equivalent nonpolynomial Lagrangian of the PST form [20]. In order to see that, we solve the algebraic equation of motion for the field $R_{\mu_{1} \ldots \mu_{p}}$,

$$
\mathcal{F}_{\mu_{1} \ldots \mu_{p+1}} c^{\mu_{1}}-c^{2} R_{\mu_{2} \ldots \mu_{p+1}}+(-1)^{p+1} p c_{\left[\mu_{2}\right.} R_{\left.\mu_{3} \ldots \mu_{p+1}\right] \mu_{1}} c^{\mu_{1}}=0 .
$$

Due to an (algebraic!) gauge symmetry of the Lagrangian (2.1), given via

$$
\delta R_{\mu_{1} \ldots \mu_{p}}=c_{\left[\mu_{1}\right.} \lambda_{\left.\mu_{2} \ldots \mu_{p}\right]},
$$

the solution is fixed only up to an arbitrary $(p-1)$-form field $\lambda_{\mu_{1} \ldots \mu_{p-1}}$ :

$$
R_{\mu_{1} \ldots \mu_{p}}=\frac{1}{c^{2}} \mathcal{F}_{\nu \mu_{1} \ldots \mu_{p}} c^{\nu}+c_{\left[\mu_{1}\right.} \lambda_{\left.\mu_{2} \ldots \mu_{p}\right]} .
$$

A simple way to solve for $R_{\mu_{1} \ldots \mu_{p}}$ is to choose a gauge $R_{\mu_{1} \ldots \mu_{p}} c^{\mu_{1}}=0$, then the solution is (2.6) without the last term, which reflects the gauge freedom. We can now plug back (2.6) to $(2.1)$ and arrive at the following Lagrangian:

$$
\mathcal{L}=-\frac{1}{2(p+1)} F_{\mu_{1} \ldots \mu_{p+1}} F^{\mu_{1} \ldots \mu_{p+1}}+\frac{1}{2 c^{2}} \mathcal{F}_{\mu_{1} \ldots \mu_{p} \nu} c^{\nu} \mathcal{F}^{\mu_{1} \ldots \mu_{p} \rho} c_{\rho}+G^{\mu \nu} \partial_{[\mu} c_{\nu]},
$$

It is a trivial exercise to show the equivalence of the Lagrangian (2.7) with that of [20]. It is straightforward to integrate out the Lagrange multiplier field $G^{\mu \nu}$ in both (2.1) and (2.7), solving the zero-curvature equation for $c_{\mu}$ as $c_{\mu}=\partial_{\mu} a$ and plugging back into the action. The minimal polynomial form thus contains only two auxiliary fields on top of the physical field $\varphi$ - the $p$-form $R_{\mu_{1} \ldots \mu_{p}}(x)$ and the scalar $a(x)$ :

$$
\begin{aligned}
\mathcal{L}= & -\frac{1}{2(p+1)} F_{\mu_{1} \ldots \mu_{p+1}} F^{\mu_{1} \ldots \mu_{p+1}}-\frac{1}{2(p+1)}\left(\mathcal{F}_{\mu_{1} \ldots \mu_{p+1}}-(p+1) \partial_{\left[\mu_{1}\right.} a R_{\left.\mu_{2} \ldots \mu_{p+1}\right]}\right) \\
& \times\left(\mathcal{F}^{\mu_{1} \ldots \mu_{p+1}}-(p+1) \partial^{\left[\mu_{1}\right.} a R^{\left.\mu_{2} \ldots \mu_{p+1}\right]}\right),
\end{aligned}
$$

Integrating out $G^{\mu \nu}$ in (2.7), we get the familiar PST form. This step involves solving a differential equation: the two actions are not guaranteed to admit equivalent interactions.

Note, that $\mathcal{F}^{2}=\mathcal{F} \wedge \mathcal{F}=0$. In fact, the simplest polynomial extension for the PST Lagrangian (2.7) would be the following one:

$$
\begin{aligned}
\mathcal{L}= & -\frac{1}{2(p+1)} F_{\mu_{1} \ldots \mu_{p+1}} F^{\mu_{1} \ldots \mu_{p+1}}-\frac{1}{2} \mathcal{F}_{\mu_{1} \ldots \mu_{p+1}} c^{\mu_{1}} R^{\mu_{2} \ldots \mu_{p+1}} \\
& +\frac{1}{4} c^{2} R^{\mu_{1} \ldots \mu_{p}} R_{\mu_{1} \ldots \mu_{p}}+G^{\mu \nu} \partial_{[\mu} c_{\nu]}
\end{aligned}
$$

which differs from (2.1) by a term $\left(R_{\mu_{1} \ldots \mu_{p}} c^{\mu_{p}}\right)^{2}$, essential for the gauge symmetry (2.5). Addition of that term promotes the second class constraint $R_{\mu_{1} \ldots \mu_{p}} c^{\mu_{p}}=0$ to first class: while in (2.9) it is a consequence of the equations of motion (similar to divergence-free condition $\partial^{\mu} A_{\mu}=0$ of the vector field in Proca theory), in (2.1) it is a gauge choice (analogous to the Lorentz gauge condition $\partial^{\mu} A_{\mu}=0$ in Maxwell theory). 


\subsection{Manifestation of two abelian gauge symmetries}

The Lagrangian (2.8) can be recast in the following form:

$$
\begin{aligned}
\mathcal{L}= & -\frac{1}{2(p+1)}\left(F_{\mu_{1} \ldots \mu_{p+1}}-(p+1) \partial_{\left[\mu_{1}\right.} a R_{\left.\mu_{2} \ldots \mu_{p+1}\right]}\right)\left(F^{\mu_{1} \ldots \mu_{p+1}}-(p+1) \partial^{\left[\mu_{1}\right.} a R^{\left.\mu_{2} \ldots \mu_{p+1}\right]}\right) \\
& +\frac{1}{(p+1) !} \epsilon_{\mu_{1} \ldots \mu_{p+1} \nu_{1} \ldots \nu_{p+1}} F^{\nu_{1} \ldots \nu_{p+1}} \partial^{\mu_{1}} a R^{\mu_{2} \ldots \mu_{p+1}}
\end{aligned}
$$

which, after a field redefinition $\varphi_{\mu_{1} \ldots \mu_{p}} \rightarrow \varphi_{\mu_{1} \ldots \mu_{p}}+a R_{\mu_{1} \ldots \mu_{p}}$ can be rewritten in the form:

$$
\begin{aligned}
\mathcal{L}= & -\frac{1}{2(p+1)}\left(F_{\mu_{1} \ldots \mu_{p+1}}+a Q_{\mu_{1} \ldots \mu_{p+1}}\right)\left(F^{\mu_{1} \ldots \mu_{p+1}}+a Q^{\mu_{1} \ldots \mu_{p+1}}\right) \\
& -\frac{1}{(p+1)(p+1) !} \epsilon_{\mu_{1} \ldots \mu_{p+1} \nu_{1} \ldots \nu_{p+1}} a F^{\nu_{1} \ldots \nu_{p+1}} Q^{\mu_{1} \ldots \mu_{p+1}},
\end{aligned}
$$

where $Q_{\mu_{1} \ldots \mu_{p+1}}=(p+1) \partial_{\left[\mu_{1}\right.} R_{\left.\mu_{2} \ldots \mu_{p+1}\right]}$. The form of the action (2.11) manifests two abelian gauge symmetries of the $p$-forms $\varphi_{\mu_{1} \ldots \mu_{p}}$ and $R_{\mu_{1} \ldots \mu_{p}}$. At the same time, similarly to the original action (2.1), fixing constant $a(2.11)$ gives a single non-chiral $p$-form action. This discontinuity is tracked also in the fact that the PST form of the action (2.7) is singular for the constant $a$ or other configurations with $c_{\mu} c^{\mu}=\partial_{\mu} a \partial^{\mu} a=0$. Finally, one can rewrite the action in the form, resembling (2.8):

$$
\begin{aligned}
\mathcal{L}= & -\frac{1}{2(p+1)} F_{\mu_{1} \ldots \mu_{p+1}} F^{\mu_{1} \ldots \mu_{p+1}} \\
& -\frac{1}{2(p+1)}\left(\mathcal{F}_{\mu_{1} \ldots \mu_{p+1}}+a Q_{\mu_{1} \ldots \mu_{p+1}}\right)\left(\mathcal{F}^{\mu_{1} \ldots \mu_{p+1}}+a Q^{\mu_{1} \ldots \mu_{p+1}}\right),
\end{aligned}
$$

Combining the equations of motion $E^{\varphi}, E^{R}$ for the fields $\varphi_{\mu_{1} \ldots \mu_{p}}$ and $R_{\mu_{1} \ldots \mu_{p}}$ one gets ${ }^{3}$

$$
\begin{gathered}
E_{\mu_{2} \ldots \mu_{p+1}}^{R}+a E_{\mu_{2} \ldots \mu_{p+1}}^{\varphi}=\partial^{\mu_{1}} a P_{\mu_{1} \ldots \mu_{p+1}}=0 \\
P_{\mu_{1} \ldots \mu_{p+1}} \equiv \mathcal{F}_{\mu_{1} \ldots \mu_{p+1}}+a Q_{\mu_{1} \ldots \mu_{p+1}}^{+}
\end{gathered}
$$

which implies

$$
P_{\mu_{1} \ldots \mu_{p+1}}=0,
$$

automatically satisfying the equation of motion $E^{a}$ for the $a$ field,

$$
E^{a}=Q_{\mu_{1} \ldots \mu_{p+1}} P^{\mu_{1} \ldots \mu_{p+1}}=0 .
$$

This indicates the existence of a PST like symmetry shifting the scalar field $a$ which should have been expected given the equivalence to the PST formulation.

\footnotetext{
${ }^{3}$ We use the notation:

$$
Q_{\mu_{1} \ldots \mu_{p+1}}^{ \pm}=Q_{\mu_{1} \ldots \mu_{p+1}} \pm \frac{1}{(p+1) !} \epsilon_{\mu_{1} \ldots \mu_{p+1} \nu_{1} \ldots \nu_{p+1}} Q^{\nu_{1} \ldots \nu_{p+1}}
$$

for (anti)self-dual part of the $(p+1)$-form $Q_{\mu_{1} \ldots \mu_{p+1}}$.
} 
An interesting generalisation of the Lagrangian (2.11) is (suppressing Lorentz indices):

$$
\mathcal{L}=-\frac{1}{2} f(a)\left(\sqrt{a} F+\frac{1}{\sqrt{a}} Q\right)^{2}+f(a) F \wedge Q .
$$

For $f(a) \sim 1 / a$, this Lagrangian is equivalent to (2.11) and describes a single chiral p-form carried in field $\varphi$. For $f(a) \sim a$, it describes an anti-chiral $p$-form field carried by $R$. The exchange $\varphi \leftrightarrow R, a \rightarrow-\frac{1}{a}, f(a) \rightarrow-f(a)$ is a symmetry of the Lagrangian (2.17).

The Lagrangian (2.11) can be also rewritten as: ${ }^{4}$

$$
\mathcal{L}=-\frac{p !}{2}\left(\mathcal{M}_{I J} F^{I} \wedge \star F^{J}+\mathcal{K}_{I J} F^{I} \wedge F^{J}\right),
$$

with

$$
\mathcal{M}_{I J}=\left[\begin{array}{cc}
1 & a \\
a & a^{2}
\end{array}\right], \quad \mathcal{K}_{I J}=\left[\begin{array}{cc}
0 & a \\
-a & 0
\end{array}\right], \quad F^{I}=\left[\begin{array}{c}
F \\
Q
\end{array}\right]
$$

where $F^{I}$ is a two-vector with $p+1$-form components, $\mathcal{M}$ is a two-by-two matrix of rank one, while the "background matrix" $\mathcal{E}=\mathcal{M}+\mathcal{K}$ is invertible. The same action with the inverted background matrix $\mathcal{E}^{-1}$ describes the same degrees of freedom, exchanging the roles of p-forms $\varphi$ and $R$. The inversion of the background matrix is a particular $\operatorname{sl}(2, R)$ rotation of the two-vector $F^{I}$ and therefore a field redefinition. A potential generalisation of (2.18) to $N$ chiral $p$-forms would be extending the matrices $\mathcal{M}_{I J}$ and $\mathcal{K}_{I J}$ to $2 N \times 2 N$ matrices, where $\mathcal{M}_{I J}$ has rank $N$. The Lagrangian (2.11) is self-dual with respect to dualisation of both $\varphi$ and $R$ fields. The replacement of the field $\varphi$ with its magnetic dual $\tilde{\varphi}$ via $F=d \varphi=\star d \tilde{\varphi}$ renders the same Lagrangian with $\varphi$ replaced by $\tilde{\varphi}$. Same is true for the replacement $Q=d R=\star d \tilde{R}$.

Next we will take a closer look at particular examples in different dimensions.

\section{Chiral two-form in six dimensions}

The first example we consider is the chiral two-form in six dimensions. For that we introduce two-forms $B_{\mu \nu}, R_{\mu \nu}$ and a vector field $c_{\mu}$, as well as a Lagrange multiplier $G^{\mu \nu}$. The Lagrangian (2.1) is given in the following form:

$$
\mathcal{L}=-\frac{1}{6} H_{\mu \nu \lambda} H^{\mu \nu \lambda}-\frac{1}{6}\left(\mathcal{F}_{\mu \nu \lambda}-3 c_{[\mu} R_{\nu \lambda]}\right)\left(\mathcal{F}^{\mu \nu \lambda}-3 c^{[\mu} R^{\nu \lambda]}\right)+G^{\mu \nu} \partial_{[\mu} c_{\nu]},
$$

where we denote:

$$
\mathcal{F}_{\mu \nu \lambda}=H_{\mu \nu \lambda}+\frac{1}{6} \epsilon_{\mu \nu \lambda \alpha \beta \gamma} H^{\alpha \beta \gamma}, \quad H_{\mu \nu \lambda}=3 \partial_{[\mu} B_{\nu \lambda]} .
$$

The property of self-duality:

$$
\mathcal{F}_{\mu \nu \lambda}=\frac{1}{6} \epsilon_{\mu \nu \lambda \alpha \beta \gamma} \mathcal{F}^{\alpha \beta \gamma},
$$

\footnotetext{
${ }^{4} \star F$ is the Hodge dual of $F$ and $\wedge$ denotes the exterior product of forms.
} 
implies:

$$
\mathcal{F}_{\mu \nu \lambda} \mathcal{F}^{\mu \nu \lambda}=0=\mathcal{F}_{[\mu \nu \lambda} \mathcal{F}_{\alpha \beta \gamma]} \cdot
$$

Integrating out from the action (3.1) the auxiliary field $R_{\mu \nu}$ by solving its equations of motion,

$$
\mathcal{F}_{\mu \nu \lambda} c^{\lambda}-c^{2} R_{\mu \nu}-2 c_{[\mu} R_{\nu] \lambda} c^{\lambda}=0 \quad \rightarrow \quad R_{\mu \nu}=\frac{1}{c^{2}} \mathcal{F}_{\mu \nu \lambda} c^{\lambda}+c_{[\mu} \lambda_{\nu]}
$$

one gets PST Lagrangian (up to total derivatives),

$$
\mathcal{L}=-\frac{1}{6} H_{\mu \nu \lambda} H^{\mu \nu \lambda}+\frac{1}{2 c^{2}} \mathcal{F}_{\mu \nu \lambda} c^{\lambda} \mathcal{F}^{\mu \nu \rho} c_{\rho}+G^{\mu \nu} \partial_{\mu} c_{\nu},
$$

which describes a free anti-self-dual two-form. The gauge symmetries of the Lagrangian (3.6) can be extended to (3.1) by assigning appropriate gauge transformations for the new auxiliary field $R_{\mu \nu}$. One of the symmetries of this system are given as (A.16):

$$
\begin{aligned}
& \delta_{\alpha} B_{\mu \nu}=c_{[\mu} \alpha_{\nu]}, \quad \delta_{\alpha} G^{\mu \nu}=\epsilon^{\mu \nu \lambda \alpha \beta \gamma} \alpha_{\lambda} c_{\alpha} R_{\beta \gamma}, \quad \delta_{\alpha} c_{\mu}=0, \\
& \delta_{\alpha} R_{\mu \nu}=-\partial_{[\mu} \alpha_{\nu]}+\frac{3}{2 c^{2}} \Pi_{-\mu \nu \lambda}{ }^{\alpha \beta \gamma} c^{\lambda} \partial_{\alpha} c_{\beta} \alpha_{\gamma} .
\end{aligned}
$$

It is straightforward to see, that the Lagrangian (3.1) and gauge transformations (3.7) simplify for a choice of a background value for the pure gauge field $c_{\mu}=\delta_{\mu}^{6}$. Nevertheless, it is useful to keep manifest Lorentz symmetry as realised in (3.1). Another symmetry of the Lagrangian (3.1) is given as (see appendix A for details):

$$
\begin{aligned}
\delta_{\varphi} c_{\mu} & =\partial_{\mu} \varphi, \quad \delta_{\varphi} B_{\mu \nu}=\varphi R_{\mu \nu}, \quad \delta_{\varphi} G^{\mu \nu}=-\frac{1}{2} \varphi \epsilon^{\mu \nu \lambda \rho \alpha \beta} R_{\lambda \rho} R_{\alpha \beta}, \\
\delta_{\varphi} R_{\mu \nu} & =\frac{3}{2 c^{2}} \varphi c^{\lambda} \Pi_{-\mu \nu \lambda}{ }^{\alpha \beta \gamma} \partial_{\alpha} R_{\beta \gamma} .
\end{aligned}
$$

Another symmetry of the action (3.1) is given by a transformation of $R_{\mu \nu}$ field only:

$$
\delta_{\phi} R_{\mu \nu}=c_{[\mu} \phi_{\nu]}
$$

These symmetries are finite-step reducible. For example, choosing:

$$
\alpha_{\mu}=c_{\mu} \phi, \quad \phi_{\mu}=\partial_{\mu} \phi
$$

we get

$$
\delta_{\alpha} B_{\mu \nu}=0, \quad \delta_{\alpha} G^{\mu \nu}=0, \quad \delta_{\alpha+\phi} R_{\mu \nu} \approx 0,
$$

where the last identity holds on $G^{\mu \nu}$-shell.

Any consistent non-abelian extension of (3.1) is expected to possess same number of symmetries as the abelian action, deforming these symmetry transformations by terms proportional to coupling constants of non-abelian interactions. 


\section{Chiral boson in two dimensions}

In two space-time dimensions of Minkowski signature, one can define the polynomial action for the free Chiral boson (2.1) in the following form:

$$
S_{N e w}=\int\left(-\frac{1}{2} \partial_{\mu} \varphi \partial^{\mu} \varphi-\frac{1}{2}\left(\mathcal{F}^{\mu}-c^{\mu} R\right)\left(\mathcal{F}_{\mu}-c_{\mu} R\right)+\lambda \epsilon^{\mu \nu} \partial_{\mu} c_{\nu}\right) d^{2} x,
$$

where we introduce notations

$$
\mathcal{F}_{\mu}=\partial_{\mu}^{+} \varphi, \quad \partial_{\mu}^{ \pm}=\partial_{\mu} \pm \epsilon_{\mu \nu} \partial^{\nu} .
$$

The field $R$ is auxiliary, and can be integrated out, solving its algebraic equation, which gives:

$$
R=\frac{1}{c^{2}} c^{\mu} \mathcal{F}_{\mu},
$$

Plugging this back in the action, we get Pasti-Sorokin-Tonin form of the action:

$$
S_{\mathrm{PST}}=\int\left(-\frac{1}{2} \partial_{\mu} \varphi \partial^{\mu} \varphi+\frac{1}{2 c^{2}} c^{\mu} \mathcal{F}_{\mu} c^{\nu} \mathcal{F}_{\nu}+\lambda \epsilon^{\mu \nu} \partial_{\mu} c_{\nu}\right) d^{2} x
$$

The latter action has the following gauge symmetry:

$$
\delta c_{\mu}=\partial_{\mu} \alpha, \quad \delta \varphi=\alpha \frac{1}{c^{2}} c^{\mu} \mathcal{F}_{\mu}, \quad \delta \lambda=\alpha\left(\frac{c^{\mu} \mathcal{F}_{\mu}}{c^{2}}\right)^{2},
$$

which can be shown by direct computation, using the identity $\mathcal{F}_{\mu}=\epsilon_{\mu \nu} \mathcal{F}^{\nu}$.

One can pull back the symmetry (4.5) to the polynomial action (4.1) (as explained, e.g., in [34] (exercise 3.17)). The difference between the actions (4.1) and (4.4) is given as:

$$
S_{\mathrm{New}}-S_{\mathrm{PST}}=\int\left(-\frac{1}{2 c^{2}}\left(c^{\mu} \mathcal{F}_{\mu}-c^{2} R\right)^{2}\right) d^{2} x,
$$

and involves the square of the equations of motion for the field $R$, as expected. Therefore, the gauge transformation (4.5) of the new action can be set to zero by assigning a gauge transformation rule for the field $R$. One can also use the equations of the auxiliary field to simplify gauge transformations. For the action (4.1), one can recast the symmetry as:

$$
\delta c_{\mu}=\partial_{\mu} \alpha, \quad \delta \varphi=\alpha R, \quad \delta \lambda=\alpha R^{2}, \quad \delta R=\alpha \frac{1}{c^{2}} c^{\mu} \partial_{\mu}^{-} R .
$$

The action (4.1) is polynomial, and among the gauge transformations (4.7) only that of the new auxiliary field $R$ is non-polynomial. In order to show that the action (4.1) is gauge invariant with respect to transformations (4.7) it is helpful to make use of the identity:

$$
\mathcal{F}_{\mu}=\frac{1}{c^{2}}\left(\epsilon_{\mu \nu} c^{\nu}+c_{\mu}\right) c^{\lambda} \mathcal{F}_{\lambda} .
$$

One can integrate out the $c_{\mu}$ field in (4.1), solving its equation of motion algebraically:

$$
c_{\mu}=\frac{1}{R} \mathcal{F}_{\mu}+\frac{1}{R^{2}} \epsilon_{\mu \nu} \partial^{\nu} \tilde{r}
$$


and plugging back into action (renaming $\frac{1}{R} \rightarrow r$ ) to get:

$$
S=\int\left(-\frac{1}{2} \partial_{\mu} \varphi \partial^{\mu} \varphi-\frac{1}{2} r^{2} \partial_{\mu} \tilde{r} \partial^{\mu} \tilde{r}-r \mathcal{F}^{\mu} \partial_{\mu} \tilde{r}\right) d^{2} x
$$

It is now straightforward to see that integrating out $r$ gives PST action (4.4), with $c_{\mu}=\partial_{\mu} \tilde{r}$ :

$$
S=\int\left(-\frac{1}{2} \partial_{\mu} \varphi \partial^{\mu} \varphi+\frac{1}{2 \partial_{\mu} \tilde{r} \partial^{\mu} \tilde{r}}\left(\mathcal{F}^{\mu} \partial_{\mu} \tilde{r}\right)^{2}\right) d^{2} x
$$

One can also arrive to this action by integrating out the Lagrange multiplier $\lambda$ in (4.4).

The action (4.10) can be written in the (2.11) form:

$$
\mathcal{S}=\int\left(-\frac{1}{2}\left(\partial_{\mu} \varphi+r \partial_{\mu} \tilde{r}\right)^{2}+\epsilon^{\mu \nu} \partial_{\mu} \varphi r \partial_{\nu} \tilde{r}\right) d^{2} x
$$

Note, that the coefficient between the two terms in the action (4.12) is fixed. Changing the sign of the second term will change the chirality of the only excitation, while any other coefficient will result in a theory with both chiral and anti-chiral degrees of freedom.

The Lagrangian of (4.12) has a discreet symmetry:

$$
r \rightarrow \tilde{r}, \quad \tilde{r} \rightarrow r, \quad \varphi \rightarrow-\varphi-r \tilde{r} .
$$

Further generalisations of chiral/duality-symmetric scalar field in two dimensions are discussed in appendix B.

\section{Duality-symmetric electromagnetism in four dimensions}

Similarly to the scalar in $2 \mathrm{~d}$ and two-form in $6 \mathrm{~d}$, one can write a duality-symmetric action of polynomial form for Maxwell field in four dimensional Minkowski space. The latter case requires doubled field content, similarly to its PST equivalent [16]. The polynomial Lagrangian for this case will be given as:

$$
\mathcal{L}=-\frac{1}{8} F_{\mu \nu}^{a} F^{a \mu \nu}-\frac{1}{8}\left(\mathcal{F}_{\mu \nu}^{a}-2 c_{[\mu} R_{\nu]}^{a}\right)\left(\mathcal{F}^{a \mu \nu}-2 c^{[\mu} R^{a \nu]}\right)+G^{\mu \nu} \partial_{[\mu} c_{\nu]},
$$

where $a, b=1,2$, and

$$
\begin{aligned}
& F_{\mu \nu}^{a}=\partial_{\mu} A_{\nu}^{a}-\partial_{\nu} A_{\mu}^{a}, \quad \mathcal{F}_{\mu \nu}^{a}=F_{\mu \nu}^{a}-\frac{1}{2} \epsilon_{a b} \varepsilon_{\mu \nu \lambda \rho} F^{b \lambda \rho}, \\
& \epsilon_{a b}=-\epsilon_{b a}, \quad \epsilon_{12}=1=\epsilon^{12}, \quad \varepsilon_{0123}=1=-\varepsilon^{0123} \text {. }
\end{aligned}
$$

The following identities hold (Einstein summation rule is assumed for both types of indices):

$$
\mathcal{F}_{\mu \nu}^{a} \mathcal{F}^{a \mu \nu}=0, \quad \varepsilon_{\mu \nu \lambda \rho} \mathcal{F}^{a \lambda \rho}=2 \epsilon^{a b} \mathcal{F}_{\mu \nu}^{b},
$$

Solving the algebraic equations of motion for $R_{\mu}^{a}$, we get the PST action:

$$
\mathcal{L}_{\mathrm{PST}}=-\frac{1}{8} F_{\mu \nu}^{a} F^{a \mu \nu}+\frac{1}{4 c_{\mu} c^{\mu}} \mathcal{F}_{\mu \nu}^{a} c^{\nu} \mathcal{F}^{a \mu \rho} c_{\rho}+G^{\mu \nu} \partial_{[\mu} c_{\nu]} .
$$


The analogue of (2.12) in this case would be the following Lagrangian

$$
\mathcal{L}=-\frac{1}{8} F_{\mu \nu}^{a} F^{a \mu \nu}-\frac{1}{8}\left(\mathcal{F}_{\mu \nu}^{a}+a Q_{\mu \nu}^{a}\right)\left(\mathcal{F}^{a \mu \nu}+a Q^{a \mu \nu}\right)
$$

where $Q_{\mu \nu}^{a}=\partial_{\mu} R_{\nu}^{a}-\partial_{\nu} R_{\mu}^{a}$. This Lagrangian describes a single Maxwell field, using four vectors and a scalar. It can be also written in the form, similar to (2.18):

$$
\mathcal{L}=-\frac{1}{8} \mathcal{M}_{I J} F_{\mu \nu}^{I} F^{J \mu \nu}-\frac{1}{16} \mathcal{K}_{I J} \epsilon^{\mu \nu \alpha \beta} F_{\mu \nu}^{I} F_{\alpha \beta}^{J},
$$

where

$$
\mathcal{M}_{I J}=\left[\begin{array}{cccc}
1 & 0 & a & 0 \\
0 & 1 & 0 & a \\
a & 0 & a^{2} & 0 \\
0 & a & 0 & a^{2}
\end{array}\right], \quad \mathcal{K}_{I J}=\left[\begin{array}{cccc}
0 & 0 & 0 & a \\
0 & 0 & -a & 0 \\
0 & -a & 0 & 0 \\
a & 0 & 0 & 0
\end{array}\right], \quad F^{I}=\left[\begin{array}{c}
F^{1} \\
F^{2} \\
Q^{1} \\
Q^{2}
\end{array}\right] .
$$

Field redefinitions can lead to different matrices $\mathcal{M}$ and $\mathcal{K}$. The detailed study of the rich symmetries of this Lagrangian will be conducted elsewhere.

\section{Conclusions}

In this work we have constructed a polynomial action for free chiral $p$-forms with manifest Lorentz symmetry, finite number of auxiliary fields and consistent with general covariance, contrary to the folklore scepticism about the possibility of such a formulation. We showed that it reproduces the non-polynomial action formulation by Pasti, Sorokin and Tonin upon gauge fixing and integrating out an auxiliary field. An interesting feature of this formalism is that it is available only in Lorentzian signature. The covariant actions found here have rich structure of symmetries, which will be studied in detail elsewhere. Seemingly conventional form of the action (2.18) encourages to study more systematically kinetic terms with non-invertible field-dependent bilinear forms. This may open a Pandora box of a large number of unexplored possibilities.

The actions presented here can be reduced to the non-covariant formulations of $[7,9,13]$ upon gauge-fixing and integrating out auxiliary fields. The latter statement is true already for the PST formulation, therefore the equivalence to PST formulation makes it evident. The dualization properties of the actions presented here are somewhat similar to those of PST actions (see, e.g., [22]), therefore there could be an alternative formulation of the polynomial actions (2.1) and (2.12) where the last term with Lagrange multiplier is replaced by $\lambda \partial^{\mu} c_{\mu}$. Then, the divergence of $c_{\mu}$ (instead of the curl) is constrained to be zero by the e.o.m. of the Lagrange multiplier, implying that $c_{\mu}$ is a dual curvature of a $(d-2)$-form field, thus reproducing an alternative action (covariantisation of Zwanziger-type action [1] instead of the Schwarz-Sen one [13]) for the chiral $p$-form.

The formulation we derive here adds an auxiliary field, another $p$-form, on top of the minimal PST formulation. It can be therefore described as "doubling $p$-forms to describe half a $p$-form". The new formulation is related to PST by a variant of Hubbard-Stratonovich transformation, up to subtleties related to gauge fixing. As discussed after eq. (2.18), there 
are non-trivial duality symmetries relating the "physical" field $\varphi$ and "auxiliary" field $R$. Therefore, we have reasons to expect that this formulation is more than a polynomial rewriting of the PST action. In any case, the usefulness of this formalism will be tested by its ability to capture non-trivial interactions. This is a work in progress [35].

The problem of interactions for chiral $p$-forms (and $p$-forms in general) has a long history [36-51] with large body of negative results about their non-abelian interactions (see, however, [52-60]). We hope, that the new formulation of the free theory presented here may help in the problem of interactions. It has two (arguable) advantages compared to the Sen's formulation [32, 33] - general covariance and formulation in terms of gauge potentials. Indeed, the gravitational coupling of the chiral $p$-forms are automatically consistent [46, 61], if there are no additional degrees of freedom in the theory, like in [32, 33]. Therefore, the actions (2.1) and (2.12) can be promoted to a generally covariant ones replacing the Minkowski metric with the dynamical metric of Einstein gravity.

One immediate question that can be asked is whether the formulation of the chiral bosons described here is advantageous compared to the PST formulation. The first challenge in this direction would be to formulate the non-linear DBI action for a single M5 brane $[38-42,62,63]$ in the variables of $(2.1)$ or (2.12). Another interesting problem would be the BRST quantisation in the lines of [61]. The actions studied here can be also used to write a Lorentz and generally covariant polynomial action for $d=10$ Type IIB Supergravity using finitely many auxiliary fields, extending the results of $[64,65]$. Some results on interacting theories generalising the free actions presented here will be reported in [35].

\section{Acknowledgments}

The author thanks Arkady Tseytlin for stimulating discussions that motivated this work and multiple helpful suggestions. Useful discussions with Zhirayr Avetisyan, Dmitry Bykov, Franz Ciceri, Chand Devchand, Oleg Evnin, Euihun Joung, Axel Kleinschmidt, Stefan Theisen and, especially, Cedric Troessaert are also gratefully acknowledged. The author thanks Dmitri Sorokin for his detailed feedback on the draft and suggestions. This work was partially supported by Alexander von Humboldt Foundation. The author thanks Erwin Schrödinger Institute in Vienna for hospitality during the Workshop "Higher spins and holography" and Imperial College London for hospitality during several visits in the process of this work.

\section{A Abelian chiral two-forms in six dimensions}

We detail here some formulas related to the symmetries of the action (3.1). Part of the gauge transformations for this action are given as abelian gauge transformations of the $B_{\mu \nu}$ and $G^{\mu \nu}$,

$$
\delta_{\xi} B_{\mu \nu}=\partial_{\mu} \xi_{\nu}-\partial_{\nu} \xi_{\mu}, \quad \delta_{\xi} G^{\mu \nu}=\partial_{\rho} \xi^{\mu \nu \rho},
$$

where $\xi^{\mu \nu \rho}$ is an antisymmetric tensor parameter. There is another gauge symmetry of PST action, that should have a counterpart here. Transformations with respect to this 
symmetry for $B_{\mu \nu}, c_{\mu}$ and $G^{\mu \nu}$ fields takes the following form:

$$
\delta_{\alpha} B_{\mu \nu}=c_{[\mu} \alpha_{\nu]}, \quad \delta_{\alpha} G^{\mu \nu}=\epsilon^{\mu \nu \lambda \alpha \beta \gamma} \alpha_{\lambda} c_{\alpha} R_{\beta \gamma}, \quad \delta_{\alpha} c_{\mu}=0,
$$

while for the new field $R_{\mu \nu}$, as we will see, it will take the following form:

$$
\delta_{\alpha} R_{\mu \nu}=-\partial_{[\mu} \alpha_{\nu]}
$$

up to some trivial transformations that vanish on-shell.

In order to check this gauge symmetry, we derive the field equations for each field separately:

$$
\begin{aligned}
\frac{\delta \mathcal{L}}{\delta B_{\mu \nu}} & =\partial_{\lambda} H^{\mu \nu \lambda}-3 \partial_{\lambda}\left(c^{[\mu} R^{\nu \lambda]}-\frac{1}{6} \epsilon^{\mu \nu \lambda \alpha \beta \gamma} c_{\alpha} R_{\beta \gamma}\right) \\
\frac{\delta \mathcal{L}}{\delta G^{\mu \nu}} & =\partial_{[\mu} c_{\nu]}, \quad \frac{\delta \mathcal{L}}{\delta R_{\mu \nu}}=\mathcal{F}^{\mu \nu \lambda} c_{\lambda}-c^{2} R^{\mu \nu}-2 c^{[\mu} R^{\nu] \lambda} c_{\lambda}, \\
\frac{\delta \mathcal{L}}{\delta c_{\mu}} & =\mathcal{F}^{\mu \nu \lambda} R_{\nu \lambda}-c^{\mu} R^{\nu \lambda} R_{\nu \lambda}-2 c^{\nu} R^{\lambda \mu} R_{\nu \lambda}-\partial_{\rho} G^{\rho \mu}
\end{aligned}
$$

Now, we compute variations with respect to symmetries (A.2):

$$
\begin{aligned}
\frac{\delta \mathcal{L}}{\delta B_{\mu \nu}} \delta_{\alpha} B_{\mu \nu}= & -\partial_{[\mu} c_{\nu} \alpha_{\lambda]}\left(\mathcal{F}^{\mu \nu \lambda}-6 \Pi_{-\alpha \beta \gamma}^{\mu \nu \lambda} c^{\alpha} R^{\beta \gamma}\right) \\
& +c_{\mu} \partial_{\nu} \alpha_{\lambda}\left(\mathcal{F}^{\mu \nu \lambda}-3 c^{[\mu} R^{\nu \lambda]}\right)
\end{aligned}
$$

where we define projectors to (anti-)self-dual tensors:

$$
\Pi_{ \pm}^{\mu \nu \lambda}{ }_{\alpha \beta \gamma}=\frac{1}{12}\left(\delta_{\alpha \beta \gamma}^{\mu \nu \lambda} \pm \epsilon_{\alpha \beta \gamma}^{\mu \nu \lambda}\right) .
$$

These projectors satisfy:

$$
\begin{aligned}
& \Pi_{ \pm}^{\mu \nu \lambda}{ }_{\alpha \beta \gamma}=\Pi_{\mp \alpha \beta \gamma}{ }^{\mu \nu \lambda}, \quad \Pi_{ \pm}^{\mu \nu \lambda}{ }_{\alpha \beta \gamma} \Pi_{\mp}^{\alpha \beta \gamma}{ }_{\rho \sigma \tau}=0, \\
& \Pi_{ \pm}^{\mu \nu \lambda}{ }_{\alpha \beta \gamma} \Pi_{ \pm}^{\alpha \beta \gamma} \rho \sigma \tau=\Pi_{ \pm}^{\mu \nu \lambda}{ }_{\rho \sigma \tau},
\end{aligned}
$$

Variation (A.3) with the $R_{\mu \nu}$-field is given as:

$$
\frac{\delta \mathcal{L}}{\delta R_{\mu \nu}} \delta_{\alpha} R_{\mu \nu}=-c_{\mu} \partial_{\nu} \alpha_{\lambda}\left(\mathcal{F}^{\mu \nu \lambda}-3 c^{[\mu} R^{\nu \lambda]}\right)
$$

and cancels the second term in (A.7) while the variation of the $G^{\mu \nu}$, that is supposed to compensate the first term of the r.h.s. of (A.7), is given as:

$$
\delta_{\alpha} G^{\mu \nu}=\alpha_{\lambda}\left(\mathcal{F}^{\mu \nu \lambda}-6 \Pi_{-}{ }^{\mu \nu \lambda}{ }_{\alpha \beta \gamma} c^{\alpha} R^{\beta \gamma}\right)
$$


which differs from the expression in (A.2). In order to show the equivalence of the two expressions, we make use of the following identity:

$$
\begin{aligned}
0= & c^{\rho} c_{[\rho} \epsilon_{\mu \nu \lambda \alpha \beta \gamma]} \mathcal{F}^{\alpha \beta \gamma}=6 c^{2} \mathcal{F}_{\mu \nu \lambda}-18 c^{\rho} c_{[\mu} \mathcal{F}_{\nu \lambda] \rho}-3 \epsilon_{\mu \nu \lambda \rho \alpha \beta} c^{\rho} \mathcal{F}^{\alpha \beta \gamma} c_{\gamma} \\
= & 6 c^{2} \mathcal{F}_{\mu \nu \lambda}-18 c^{2} c_{[\mu} R_{\nu \lambda]}-3 c^{2} \epsilon_{\mu \nu \lambda \alpha \beta \gamma} c^{\alpha} R^{\beta \gamma} \\
& -3 \epsilon_{\mu \nu \lambda \rho \alpha \beta} c^{\rho}\left(\mathcal{F}^{\alpha \beta \gamma}-3 c^{[\alpha} R^{\beta \gamma]}\right) c_{\gamma}-18 c_{[\mu}\left(\mathcal{F}_{\nu \lambda] \rho}-2 c_{\nu} R_{\lambda] \rho}-R_{\nu \lambda]} c_{\rho}\right) c^{\rho} \\
= & 6 c^{2}\left(\mathcal{F}_{\mu \nu \lambda}-6 \Pi_{+\mu \nu \lambda}{ }^{\alpha \beta \gamma} c_{\alpha} R_{\beta \gamma}\right)-9 \Pi_{+\mu \nu \lambda}{ }^{\alpha \beta \gamma} c_{\alpha} \frac{\delta \mathcal{L}}{\delta R^{\beta \gamma}} \\
\approx & 6 c^{2}\left(\mathcal{F}_{\mu \nu \lambda}-6 \Pi_{+\mu \nu \lambda}{ }^{\alpha \beta \gamma} c_{\alpha} R_{\beta \gamma}\right),
\end{aligned}
$$

where the last identity holds on-shell (up to equations of motion for $R_{\mu \nu}$ ). Assuming that $c^{2} \neq 0$, we deduce the following identity:

$$
\mathcal{F}_{\mu \nu \lambda} \approx 6 \Pi_{+\mu \nu \lambda}{ }^{\alpha \beta \gamma} c_{\alpha} R_{\beta \gamma} .
$$

This identity allows to rewrite the variation (A.12) as:

$$
\delta_{\alpha} G^{\mu \nu} \approx 6 \alpha_{\lambda}\left(\Pi_{+}-\Pi_{-}\right)^{\mu \nu \lambda}{ }_{\alpha \beta \gamma} c^{\alpha} R^{\beta \gamma}=\epsilon^{\mu \nu \lambda \alpha \beta \gamma} \alpha_{\lambda} c_{\alpha} R_{\beta \gamma},
$$

which is exactly same as the variation (A.2) guessed from the PST symmetries.

Since the gauge transformation of the field $G^{\mu \nu}$ was changed up to equations of motion of $R_{\mu \nu}$, it induces a modification in the gauge transformations of the latter field. We compute this change for completeness here and get:

$$
\begin{aligned}
& \delta_{\alpha} B_{\mu \nu}=c_{[\mu} \alpha_{\nu]}, \quad \delta_{\alpha} G^{\mu \nu}=\epsilon^{\mu \nu \lambda \alpha \beta \gamma} \alpha_{\lambda} c_{\alpha} R_{\beta \gamma}, \quad \delta_{\alpha} c_{\mu}=0, \\
& \delta_{\alpha} R_{\mu \nu}=-\partial_{[\mu} \alpha_{\nu]}+\frac{3}{2 c^{2}} \Pi_{-\mu \nu \lambda}^{\alpha \beta \gamma} c^{\lambda} \partial_{\alpha} c_{\beta} \alpha_{\gamma} .
\end{aligned}
$$

The last term is zero on $G^{\mu \nu}$-shell. Alternative form of these gauge transformations are given as:

$$
\begin{aligned}
& \delta_{\alpha} B_{\mu \nu}=c_{[\mu} \alpha_{\nu]}, \quad \delta_{\alpha} c_{\mu}=0, \quad \delta_{\alpha} R_{\mu \nu}=-\partial_{[\mu} \alpha_{\nu]}, \\
& \delta_{\alpha} G^{\mu \nu}=\epsilon^{\mu \nu \lambda \alpha \beta \gamma} \alpha_{\lambda} c_{\alpha} R_{\beta \gamma}+\alpha_{\lambda}\left(\mathcal{F}^{\mu \nu \lambda}-6 \Pi_{+}{ }^{\mu \nu \lambda}{ }_{\alpha \beta \gamma} c^{\alpha} R^{\beta \gamma}\right) .
\end{aligned}
$$

In this case, the last term is zero on $R_{\mu \nu}$-shell as shown in (A.13). We will use the formulation (A.16) unless otherwise specified.

There is yet another gauge symmetry of the PST action, $\varphi$-symmetry:

$$
\delta_{\varphi} c_{\mu}=\partial_{\mu} \varphi, \quad \delta_{\varphi} B_{\mu \nu}=\varphi R_{\mu \nu}
$$

In order for the action (3.1) to be gauge invariant with respect to this symmetry, one has to assign gauge transformations:

$$
\delta_{\varphi} G^{\mu \nu}=-\frac{1}{2} \varphi \epsilon^{\mu \nu \lambda \rho \alpha \beta} R_{\lambda \rho} R_{\alpha \beta}, \quad \delta_{\varphi} R_{\mu \nu}=\frac{3}{2 c^{2}} \varphi c^{\lambda} \Pi_{-\mu \nu \lambda}{ }^{\alpha \beta \gamma} \partial_{\alpha} R_{\beta \gamma} .
$$

The latter symmetry of the action can be checked by direct computation. 


\section{B (Non-)chiral scalar in two dimensions}

Here we discuss some interesting observations related to chiral boson action (4.12) and its generalisations. The Lagrangian (4.12) can be also written as:

$$
\begin{aligned}
\mathcal{L} & =-\frac{1}{2} G_{A B} \partial_{\mu} \varphi^{A} \partial^{\mu} \varphi^{B}+\frac{1}{2} B_{A B} \epsilon^{\mu \nu} \partial_{\mu} \varphi^{A} \partial_{\nu} \varphi^{B}, \\
\varphi^{A} & =(\varphi, r, \tilde{r}), \quad \varphi_{A}=\delta_{A B} \varphi^{B}, \quad A, B, C=1,2,3,
\end{aligned}
$$

where the "sigma model metric" $G_{A B}$ has rank one and does not depend on $\varphi_{1} \equiv \varphi$ :

$$
G_{A B}(\varphi)=\left[\begin{array}{ccc}
1 & -\alpha \varphi_{3} & \frac{(1+\alpha)}{2} \varphi_{2} \\
-\alpha \varphi_{3} & \alpha^{2} \varphi_{3}^{2} & -\frac{\alpha(1+\alpha)}{2} \varphi_{2} \varphi_{3} \\
\frac{(1+\alpha)}{2} \varphi_{2}-\frac{\alpha(1+\alpha)}{2} \varphi_{2} \varphi_{3} & \frac{(1+\alpha)^{2}}{4} \varphi_{2}^{2}
\end{array}\right], \quad B_{A B}=\left[\begin{array}{ccc}
0 & -\gamma \varphi_{3} & \beta \varphi_{2} \\
\gamma \varphi_{3} & 0 & -\delta \varphi_{1} \\
-\beta \varphi_{2} & \delta \varphi_{1} & 0
\end{array}\right]
$$

and $\beta+\gamma+\delta=1$. The parameters $\alpha, \beta, \gamma$ are arbitrary numbers: different choices of these numbers are related by field redefinition or boundary terms in the action.

\section{B.1 A generalisation}

A generalisation of the action (4.12) is given as in (2.17):

$$
\mathcal{L}=-\frac{1}{2} f(r)\left(\sqrt{r} \partial_{\mu} \varphi_{+}+\frac{1}{\sqrt{r}} \partial_{\mu} \varphi_{-}\right)^{2}+\epsilon^{\mu \nu} f(r) \partial_{\mu} \varphi_{+} \partial_{\nu} \varphi_{-},
$$

For $f(r)=1 / r$, this action is equivalent to (4.12) and describes a single chiral scalar carried in field $\varphi_{+}$. Instead, for $f(r)=r$, it describes an anti-chiral scalar carried by $\varphi_{-}$. The replacement $\varphi_{+} \leftrightarrow \varphi_{-}, r \rightarrow-\frac{1}{r}, f(r) \rightarrow-f(r)$ is a symmetry of (B.4).

In terms of $\varphi=\varphi_{+}+\varphi_{-}, \tilde{\varphi}=\varphi_{+}-\varphi_{-}$the special choices of $f(r)=r^{ \pm 1}$ (replacing further $r \rightarrow r^{-1}$ in the second case) give:

$$
\mathcal{L}_{ \pm}=-\frac{1}{8}\left[(r+1) \partial_{\mu} \varphi \pm(r-1) \partial_{\mu} \tilde{\varphi}\right]^{2}+\frac{1}{4} \epsilon^{\mu \nu} r \partial_{\mu} \varphi \partial_{\nu} \tilde{\varphi}
$$

where different signs correspond to different chiralities. The two actions transform into each other under $\varphi \leftrightarrow \tilde{\varphi}, r \rightarrow-r$.

\section{B.2 A duality-symmetric formulation for $2 \mathrm{~d}$ scalar}

The action (4.12) can be rewritten in a classically equivalent form:

$$
\mathcal{S}=\int\left(-\frac{1}{2}\left(\partial_{\mu} \varphi-A_{\mu}\right)^{2}+\frac{1}{2} \epsilon^{\mu \nu} \varphi F_{\mu \nu}+B^{\mu}\left(A_{\mu}-r \partial_{\mu} \tilde{\varphi}\right)\right) d^{2} x
$$

where $F_{\mu \nu}=\partial_{\mu} A_{\nu}-\partial_{\nu} A_{\mu}$ and $B$ is a Lagrange multiplier.

Dropping the last term in (B.6), one can write a simpler (quadratic) action:

$$
\mathcal{S}=\int\left(-\frac{1}{2}\left(\partial_{\mu} \varphi-A_{\mu}\right)^{2}+\frac{1}{2} \epsilon^{\mu \nu} \varphi F_{\mu \nu}\right) d^{2} x
$$


which, after integrating out $A_{\mu}$ is equivalent to free scalar field action. We can double the field content of this action, in the following way:

$$
\mathcal{S}=\int\left(-\frac{1}{4}\left(\partial_{\mu} \varphi_{+}-A_{\mu}\right)^{2}-\frac{1}{4}\left(\partial_{\mu} \varphi_{-}+A_{\mu}\right)^{2}+\frac{1}{4} \epsilon^{\mu \nu}\left(\varphi_{+}+\varphi_{-}\right) F_{\mu \nu}\right) d^{2} x
$$

This action is now gauge invariant with respect to $\delta A_{\mu}=\partial_{\mu} a(x), \delta \varphi_{ \pm}= \pm a(x)$. After integrating out the field $A_{\mu}$, we arrive to free scalar action, depending only on the field $\varphi=\varphi_{+}+\varphi_{-}$. Instead of solving the equation of motion for $A_{\mu}$, we could use the equations of motion of all fields to show, that on-shell $A_{\mu}$ is pure gauge, therefore we can fix a gauge:

$$
0=A_{\mu}=\frac{1}{2}\left(\partial_{\mu}^{+} \varphi_{+}+\partial_{\mu}^{-} \varphi_{-}\right)
$$

where the second equation is the equation of motion for the field $A_{\mu}$ with $\partial_{\mu}^{ \pm} \equiv \partial_{\mu} \pm \epsilon_{\mu \nu} \partial^{\nu}$. It follows from here, that in the (on-shell!) gauge $A_{\mu}=0$, we have:

$$
\partial_{\mu}^{+} \varphi_{+}=0, \quad \partial_{\mu}^{-} \varphi_{-}=0
$$

hence the names of the fields. The action (B.8) can be rewritten in terms of $\varphi=\varphi_{+}+\varphi_{-}$ and $\tilde{\varphi}=\varphi_{+}-\varphi_{-}$in the following form:

$$
\mathcal{L}=-\frac{1}{4}\left(\partial_{\mu} \varphi\right)^{2}-\frac{1}{4}\left(\partial_{\mu} \tilde{\varphi}\right)^{2}+\frac{1}{2} A^{\mu}\left(\partial_{\mu} \tilde{\varphi}-\epsilon_{\mu \nu} \partial^{\nu} \varphi\right)-\frac{1}{4} A_{\mu} A^{\mu}
$$

Here, the gauge symmetry is given by:

$$
\delta A_{\mu}=\partial_{\mu} a, \quad \delta \tilde{\varphi}=a .
$$

On-shell, one can show that $A_{\mu}$ is pure gauge, therefore one can choose a gauge $A_{\mu}=0$, which, together with the e.o.m. for $A_{\mu}$ :

$$
A_{\mu}=\partial_{\mu} \tilde{\varphi}-\epsilon_{\mu \nu} \partial^{\nu} \varphi
$$

implies that there is only one d.o.f. propagating, as opposed to the same action without $A^{2}$ term, which would describe two degrees of freedom (see, e.g., [9]). The on-shell equivalence does not mean one can plug back $A_{\mu}=0$ to the action. Instead, we can fix, e.g., an off-shell gauge $\tilde{\varphi}=0$ which is incompatible with $A_{\mu}=0$. The fields $\varphi$ and $\tilde{\varphi}$ do not enter the action in a completely symmetric form. In particular, there is a gauge symmetry that allows to gauge fix $\tilde{\varphi}$ to zero, but not $\varphi$. In fact, if one changes the sign in front of the $A^{2}$ term, the roles of the fields $\varphi$ and $\tilde{\varphi}$ change. This is not a field redefinition though and therefore is not a symmetry of the action. We can achieve the change of the sign in that term by a redefinition: $A_{\mu} \rightarrow \epsilon_{\mu \nu} A^{\nu}$, but that results in simultaneous exchange of $\varphi$ and $\tilde{\varphi}$ in the mixed term, therefore leaving the roles of $\varphi$ and $\tilde{\varphi}$ unchanged. Even though the field $\tilde{\varphi}$ is pure gauge, it is essential that its kinetic term has the right sign. If we take the opposite sign keeping gauge invariance of the action in tact (which is tantamount to changing also the sign of the $A^{2}$ term), the action becomes topological with zero degrees of freedom. 
Open Access. This article is distributed under the terms of the Creative Commons Attribution License (CC-BY 4.0), which permits any use, distribution and reproduction in any medium, provided the original author(s) and source are credited.

\section{References}

[1] D. Zwanziger, Local Lagrangian quantum field theory of electric and magnetic charges, Phys. Rev. D 3 (1971) 880 [INSPIRE].

[2] S. Deser and C. Teitelboim, Duality transformations of Abelian and nonabelian gauge fields, Phys. Rev. D 13 (1976) 1592 [InSPIRE].

[3] N. Marcus and J.H. Schwarz, Field theories that have no manifestly Lorentz invariant formulation, Phys. Lett. 115B (1982) 111 [INSPIRE].

[4] W. Siegel, Manifest Lorentz invariance sometimes requires nonlinearity, Nucl. Phys. B 238 (1984) 307 [INSPIRE].

[5] A.R. Kavalov and R.L. Mkrtchian, Lagrangian of the selfduality equation and $d=10, N=2 b$ supergravity, Sov. J. Nucl. Phys. 46 (1987) 728 [INSPIRE].

[6] R. Floreanini and R. Jackiw, Selfdual fields as charge density solitons, Phys. Rev. Lett. 59 (1987) 1873 [INSPIRE].

[7] M. Henneaux and C. Teitelboim, Dynamics of chiral (selfdual) P forms, Phys. Lett. B 206 (1988) 650 [INSPIRE].

[8] K. Harada, The chiral Schwinger model in terms of chiral bosonization, Phys. Rev. Lett. 64 (1990) 139 [INSPIRE].

[9] A.A. Tseytlin, Duality symmetric formulation of string world sheet dynamics, Phys. Lett. B 242 (1990) 163 [INSPIRE].

[10] B. McClain, F. Yu and Y.S. Wu, Covariant quantization of chiral bosons and $\mathrm{OSp}(1,1 \mid 2)$ symmetry, Nucl. Phys. B 343 (1990) 689 [INSPIRE].

[11] C. Wotzasek, The Wess-Zumino term for chiral bosons, Phys. Rev. Lett. 66 (1991) 129 [INSPIRE].

[12] A.A. Tseytlin, Duality symmetric closed string theory and interacting chiral scalars, Nucl. Phys. B 350 (1991) 395 [inSPIRE].

[13] J.H. Schwarz and A. Sen, Duality symmetric actions, Nucl. Phys. B 411 (1994) 35 [hep-th/9304154] [INSPIRE].

[14] A. Khoudeir and N. Pantoja, Covariant duality symmetric actions, Phys. Rev. D 53 (1996) 5974 [hep-th/9411235] [INSPIRE].

[15] P. Pasti, D.P. Sorokin and M. Tonin, Note on manifest Lorentz and general coordinate invariance in duality symmetric models, Phys. Lett. B 352 (1995) 59 [hep-th/9503182] [INSPIRE].

[16] P. Pasti, D.P. Sorokin and M. Tonin, Duality symmetric actions with manifest space-time symmetries, Phys. Rev. D 52 (1995) R4277 [hep-th/9506109] [INSPIRE].

[17] P. Pasti, D.P. Sorokin and M. Tonin, Space-time symmetries in duality symmetric models, in the proceedings of Gauge theories, applied supersymmetry, quantum gravity, July 10-14, Leuven, Belgium (1995), hep-th/9509052 [INSPIRE]. 
[18] A.A. Tseytlin, Selfduality of Born-Infeld action and Dirichlet three-brane of type IIB superstring theory, Nucl. Phys. B 469 (1996) 51 [hep-th/9602064] [INSPIRE].

[19] F.P. Devecchi and M. Henneaux, Covariant path integral for chiral p forms, Phys. Rev. D 54 (1996) 1606 [hep-th/9603031] [INSPIRE].

[20] P. Pasti, D.P. Sorokin and M. Tonin, On Lorentz invariant actions for chiral $p$ forms, Phys. Rev. D 55 (1997) 6292 [hep-th/9611100] [INSPIRE].

[21] M. Cederwall and A. Westerberg, World volume fields, $\operatorname{SL}(2: Z)$ and duality: the type IIB three-brane, JHEP 02 (1998) 004 [hep-th/9710007] [INSPIRE].

[22] A. Maznytsia, C.R. Preitschopf and D.P. Sorokin, Duality of selfdual actions, Nucl. Phys. B 539 (1999) 438 [hep-th/9805110] [INSPIRE].

[23] P. Pasti, D.P. Sorokin and M. Tonin, Harmonics, notophs and chiral bosons, Lect. Notes Phys. 524 (1999) 97 [hep-th/9807133] [INSPIRE].

[24] M. Roček and A.A. Tseytlin, Partial breaking of global D $=4$ supersymmetry, constrained superfields and three-brane actions, Phys. Rev. D 59 (1999) 106001 [hep-th/9811232] [INSPIRE].

[25] R. Manvelyan, R. Mkrtchian and H.J.W. Muller-Kirsten, On different formulations of chiral bosons, Phys. Lett. B 453 (1999) 258 [hep-th/9901084] [INSPIRE].

[26] S.M. Kuzenko and S. Theisen, Supersymmetric duality rotations, JHEP 03 (2000) 034 [hep-th/0001068] [INSPIRE].

[27] Y.-G. Miao, R. Manvelyan and H.J.W. Mueller-Kirsten, Selfduality beyond chiral $p$ form actions, Phys. Lett. B 482 (2000) 264 [hep-th/0002060] [INSPIRE].

[28] D. Sorokin, Lagrangian description of duality-symmetric fields, NATO Sci. Ser. II 60 (2002) 365.

[29] C. Bunster and M. Henneaux, Duality invariance implies Poincaré invariance, Phys. Rev. Lett. 110 (2013) 011603 [arXiv: 1208.6302] [INSPIRE].

[30] H. Afshar, E. Esmaeili and M.M. Sheikh-Jabbari, Asymptotic symmetries in p-form theories, JHEP 05 (2018) 042 [arXiv: 1801.07752] [INSPIRE].

[31] V.I. Ogievetsky and I.V. Polubarinov, The notoph and its possible interactions, Sov. J. Nucl. Phys. 4 (1967) 156 [INSPIRE].

[32] A. Sen, Covariant action for type IIB supergravity, JHEP 07 (2016) 017 [arXiv: 1511.08220] [INSPIRE].

[33] A. Sen, Self-dual forms: action, hamiltonian and compactification, arXiv:1903.12196 [INSPIRE].

[34] M. Henneaux and C. Teitelboim, Quantization of gauge systems, Princeton University Press, Princeton U.S.A. (1992).

[35] K. Mkrtchyan, work in progress.

[36] D.Z. Freedman and P.K. Townsend, Antisymmetric tensor gauge theories and nonlinear $\sigma$-models, Nucl. Phys. B 177 (1981) 282 [INSPIRE].

[37] M. Henneaux, Uniqueness of the Freedman-Townsend interaction vertex for two form gauge fields, Phys. Lett. B 368 (1996) 83 [hep-th/9511145] [INSPIRE]. 
[38] M. Perry and J.H. Schwarz, Interacting chiral gauge fields in six-dimensions and Born-Infeld theory, Nucl. Phys. B 489 (1997) 47 [hep-th/9611065] [InSPIRE].

[39] P. Pasti, D.P. Sorokin and M. Tonin, Covariant action for a $D=11$ five-brane with the chiral field, Phys. Lett. B 398 (1997) 41 [hep-th/9701037] [INSPIRE].

[40] I.A. Bandos et al., Covariant action for the superfive-brane of M-theory, Phys. Rev. Lett. 78 (1997) 4332 [hep-th/9701149] [INSPIRE].

[41] J.H. Schwarz, Coupling a selfdual tensor to gravity in six-dimensions, Phys. Lett. B 395 (1997) 191 [hep-th/9701008] [INSPIRE].

[42] M. Aganagic, J. Park, C. Popescu and J.H. Schwarz, World volume action of the M-theory five-brane, Nucl. Phys. B 496 (1997) 191 [hep-th/9701166] [INSPIRE].

[43] S. Deser, A. Gomberoff, M. Henneaux and C. Teitelboim, Duality, selfduality, sources and charge quantization in Abelian $N$ form theories, Phys. Lett. B 400 (1997) 80 [hep-th/9702184] [INSPIRE].

[44] R. Medina and N. Berkovits, Pasti-Sorokin-Tonin actions in the presence of sources, Phys. Rev. D 56 (1997) 6388 [hep-th/9704093] [INSPIRE].

[45] M. Henneaux and B. Knaepen, All consistent interactions for exterior form gauge fields, Phys. Rev. D 56 (1997) R6076 [hep-th/9706119] [InSPIRE].

[46] X. Bekaert and M. Henneaux, Comments on chiral p forms, Int. J. Theor. Phys. 38 (1999) 1161 [hep-th/9806062] [INSPIRE].

[47] X. Bekaert, M. Henneaux and A. Sevrin, Deformations of chiral two forms in six-dimensions, Phys. Lett. B 468 (1999) 228 [hep-th/9909094] [INSPIRE].

[48] X. Bekaert, M. Henneaux and A. Sevrin, Symmetry deforming interactions of chiral p forms, Nucl. Phys. Proc. Suppl. 88 (2000) 27 [hep-th/9912077] [INSPIRE].

[49] X. Bekaert, M. Henneaux and A. Sevrin, Chiral forms and their deformations, Commun. Math. Phys. 224 (2001) 683 [hep-th/0004049] [INSPIRE].

[50] X. Bekaert and S. Cucu, Deformations of duality symmetric theories, Nucl. Phys. B 610 (2001) 433 [hep-th/0104048] [INSPIRE].

[51] I. Bandos, On Lagrangian approach to self-dual gauge fields in spacetime of nontrivial topology, JHEP 08 (2014) 048 [arXiv:1406.5185] [INSPIRE].

[52] P.-M. Ho and Y. Matsuo, M5 from M2, JHEP 06 (2008) 105 [arXiv:0804.3629] [INSPIRE].

[53] P. Pasti, I. Samsonov, D. Sorokin and M. Tonin, BLG-motivated Lagrangian formulation for the chiral two-form gauge field in D=6 and M5-branes, Phys. Rev. D 80 (2009) 086008 [arXiv:0907.4596] [INSPIRE].

[54] P.-M. Ho, K.-W. Huang and Y. Matsuo, A non-Abelian self-dual gauge theory in $5+1$ dimensions, JHEP 07 (2011) 021 [arXiv: 1104.4040] [INSPIRE].

[55] H. Samtleben, Actions for non-Abelian twisted self-duality, Nucl. Phys. B 851 (2011) 298 [arXiv: 1105.3216] [INSPIRE].

[56] C.-S. Chu and S.-L. Ko, Non-abelian action for multiple five-branes with self-dual tensors, JHEP 05 (2012) 028 [arXiv: 1203.4224] [INSPIRE].

[57] H. Samtleben, E. Sezgin and R. Wimmer, Six-dimensional superconformal couplings of non-abelian tensor and hypermultiplets, JHEP 03 (2013) 068 [arXiv:1212.5199] [INSPIRE]. 
[58] I. Bandos, H. Samtleben and D. Sorokin, Duality-symmetric actions for non-Abelian tensor fields, Phys. Rev. D 88 (2013) 025024 [arXiv: 1305.1304] [INSPIRE].

[59] K.-W. Huang, R. Roiban and A.A. Tseytlin, Self-dual 6d 2-form fields coupled to non-abelian gauge field: quantum corrections, JHEP 06 (2018) 134 [arXiv: 1804.05059] [INSPIRE].

[60] G. Buratti, K. Lechner and L. Melotti, Duality invariant self-interactions of abelian p-forms in arbitrary dimensions, JHEP 09 (2019) 022 [arXiv: 1906.07094] [INSPIRE].

[61] X. Bekaert and S. Cucu, Antifield BRST quantization of duality symmetric Maxwell theory, JHEP 01 (2001) 015 [hep-th/0010266] [INSPIRE].

[62] S.-L. Ko, D. Sorokin and P. Vanichchapongjaroen, The M5-brane action revisited, JHEP 11 (2013) 072 [arXiv: 1308.2231] [INSPIRE].

[63] S.-L. Ko and P. Vanichchapongjaroen, Towards $2+4$ formulation of M5-brane, JHEP 12 (2015) 170 [arXiv:1511.05395] [INSPIRE].

[64] G. Dall'Agata, K. Lechner and D.P. Sorokin, Covariant actions for the bosonic sector of $d=10$ IIB supergravity, Class. Quant. Grav. 14 (1997) L195 [hep-th/9707044] [INSPIRE].

[65] G. Dall'Agata, K. Lechner and M. Tonin, $D=10, N=I I B$ supergravity: Lorentz invariant actions and duality, JHEP 07 (1998) 017 [hep-th/9806140] [INSPIRE]. 